Research Article

\title{
Structural and Acoustic Responses of a Submerged Stiffened Conical Shell
}

\author{
Meixia Chen, Cong Zhang, Xiangfan Tao, and Naiqi Deng \\ School of Naval Architecture and Ocean Engineering, Huazhong University of Science and Technology, Wuhan, Hubei 430074, China \\ Correspondence should be addressed to Cong Zhang; zc_celia@163.com
}

Received 5 February 2013; Accepted 3 December 2013; Published 4 March 2014

Academic Editor: Anindya Ghoshal

Copyright ( 2014 Meixia Chen et al. This is an open access article distributed under the Creative Commons Attribution License, which permits unrestricted use, distribution, and reproduction in any medium, provided the original work is properly cited.

\begin{abstract}
This paper studies the vibrational behavior and far-field sound radiation of a submerged stiffened conical shell at low frequencies. The solution for the dynamic response of the conical shell is presented in the form of a power series. A smeared approach is used to model the ring stiffeners. Fluid loading is taken into account by dividing the conical shell into narrow strips which are considered to be local cylindrical shells. The far-field sound pressure is solved by the Element Radiation Superposition Method. Excitations in two directions are considered to simulate the loading on the surface of the conical shell. These excitations are applied along the generator and normal to the surface of the conical shell. The contributions from the individual circumferential modes on the structural responses of the conical shell are studied. The effects of the external fluid loading and stiffeners are discussed. The results from the analytical models are validated by numerical results from a fully coupled finite element/boundary element model.
\end{abstract}

\section{Introduction}

The reduction of noise emitted by maritime platforms is an important topic in naval research. A truncated cone is used to support the aft-shaft-bearing of the submarine propeller. The structural and acoustic responses of a conical shell are not as widely reported in literature as in the case of cylindrical shells. This is due to the increased mathematical complexity associated with the effect of the variation of the radius along the length of the cone on the elastic waves. Much of the earlier work on the equations of motion for conical shells and forms of solution with different boundary conditions has been summarized by Leissa [1]. The approximate locations of the natural frequencies have been found using the Rayleigh-Ritz method by several authors $[2,3]$. Tong presented a procedure for the free vibration analysis of isotropic and orthotropic conical shells in the form of a power series [4]. Guo studied the propagation and radiation properties of elastic waves in a cone in vacuo [5]. For fluid loaded conical shells, Guo investigated fluid loading effects of waves on conical shells in which a perturbation technique is applied by introducing a small vertex angle parameter [6]. Caresta and Kessissoglou considered the fluid loading by dividing the conical shell into narrow strips and each of these strips is treated as a locally cylindrical shell [7]. Donnell-Mushtari and Flügge equations of motion were compared by Caresta and Kessissoglou [8]. It was shown that in order to use the Flügge equations of motion with the power series solution, an approximation of the shear force is required.

Many researchers have investigated approximate methods to solve the acoustic radiation from vibrating elastic structures. The boundary integral equation formulation is a popular method. A good review on the evolution of boundary element techniques was given by Chien et al. [9]. Coupled finite element/boundary element methods have been presented by Jeans and Mathews [10]. Caresta and Kessissoglou used the Helmholtz integral to calculate the far-field radiated sound pressure of a submerged cylindrical shell closed by two truncated conical shells [11]. Since the boundary element method (BEM) requires significant computational time and memory, many researchers have been working on improving the efficiency of BEM [12] or exploring other approximate methods $[13,14]$. Wang et al. proposed an approximate method named Element Radiation Superposition Method (ERSM) to predict the radiated pressure based on the surface velocity [15]. The total radiation pressure equals the superposition of the radiated pressure from each surface element, which can be obtained through 


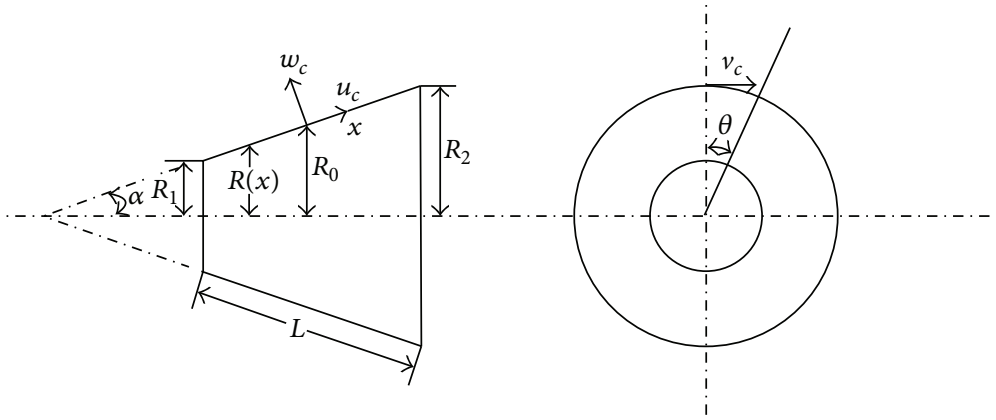

FIGURE 1: Coordinate system and displacements for a thin conical shell.

weighting the vibration velocity using the transfer quantity. The proposed method was found to be superior in terms of computational efficiency. ERSM can be implemented to solve various models by adopting regular baffles such as spherical, infinite cylindrical and spherical baffles.

In this work, the structural and acoustic responses of a stiffened conical shell submerged in a heavy fluid are presented. Section 2 describes the solution for the dynamic response of a conical shell, which is presented in the form of a power series. Fluid loading is taken into account by dividing the shell into narrow strips, similar to the procedures presented by Caresta and Kessissoglou [7]. The stiffening of the structure is considered by smearing the stiffeners over the conical shell surface, which results in orthotropic material behavior of the conical shell. In Section 3, the Element Radiation Superposition Method (ERSM) is introduced to solve the far-field pressure of a conical shell, based on the theory presented in [15]. The total acoustic pressure of conical shell is assumed to be the superposition of the radiated pressure of all divided elements, which is approximated using the analytical solution of the radiated pressure from a piston with a cylindrical baffle. In Section 4 , the validity and convergence of the method to solve the structural responses and the far-field acoustic pressure of a stiffened conical shell are discussed. The contributions from the individual circumferential modes for different load cases are examined. The effects of the external fluid loading and the ring stiffeners are described.

\section{Dynamic Model of a Submerged Stiffened Conical Shell}

2.1. Dynamic Response of a Conical Shell. For a conical shell, the coordinate system $(x, \theta)$ is defined in Figure $1 . u_{c}$ and $v_{c}$ are the orthogonal components of the displacement in the $x$ and $\theta$ directions, respectively, and $w_{c}$ is the displacement normal to the shell surface. $\alpha$ is the semivertex angle of the conical shell. $R_{0}$ is the mean radius of the shell. $R_{1}$ is the radius of the smaller end and $R_{2}$ is the radius of the larger end. $R(x)$ is the radius of the cone at location $x . L$ is the length of the cone along its generator. According to the Flügge theory, the equations of motion are given by [1]

$$
\begin{gathered}
\frac{\partial\left(R(x) N_{x}\right)}{\partial x}+\frac{\partial N_{\theta x}}{\partial \theta}-N_{\theta} \sin \alpha-R(x) \rho h \frac{\partial^{2} u_{c}}{\partial t^{2}}=0 \\
\frac{\partial N_{\theta}}{\partial \theta}+\frac{\partial\left(R(x) N_{x \theta}\right)}{\partial x}+N_{\theta x} \sin \alpha+Q_{\theta} \cos \alpha \\
-R(x) \rho h \frac{\partial^{2} v_{c}}{\partial t^{2}}=0 \\
-N_{\theta} \cos \alpha+\frac{\partial\left(R(x) Q_{x}\right)}{\partial x}+\frac{\partial Q_{\theta}}{\partial \theta}-R(x) \rho h \frac{\partial^{2} w_{c}}{\partial t^{2}}=0
\end{gathered}
$$

where $\rho$ and $h$ are the density and the thickness of the conical shell, respectively. $N_{x}, N_{\theta}, N_{\theta x}, N_{x \theta}, Q_{x}$, and $Q_{\theta}$ are the forces for a conical shell and are given in the Appendix. For a conical shell with stiffeners, the effect of the stiffeners can be considered by averaging their properties over the surface of the conicalshell. The smeared approach can be used when the shells are reinforced by closely spaced uniform stiffeners [16]. Figure 2 shows a schematic diagram of the ring stiffeners. $E_{r}$ is the modulus of elasticity of the stiffeners. $b_{r}$ and $h_{r}$ are the thickness and height of the ring stiffeners, respectively. $A=b_{r} h_{r}$ is the cross-sectional area of the ring stiffeners. $e$ is the distance from the centroid of the stiffener to the shell. $I$ is the moment of inertia of the stiffeners about line of the reference (the middle line of the shell). $d$ and $d_{L}=d / \cos \alpha$ are the distances between the ring stiffeners along the axial and generator direction of the conical shell, respectively. Using the smeared approach, the thickness of the conical shell, $h$, in (2.1) is replaced by $\bar{h}$, where $\bar{h}=h+A / d_{L} \cdot N_{\theta}$ and $M_{\theta}$ are replaced by $N_{\theta}^{\prime}$ and $M_{\theta}^{\prime}$, where [17]

$$
\begin{aligned}
& N_{\theta}^{\prime}=N_{\theta}+\mu \varepsilon_{\theta}+\chi k_{\theta}, \\
& M_{\theta}^{\prime}=M_{\theta}+\chi \varepsilon_{\theta}+\eta k_{\theta}
\end{aligned}
$$

and $\varepsilon_{\theta}$ is the strain in the shell and $k_{\theta}$ is the variation of the curvature. Consider $\mu=E_{r} A / d_{L},=E_{r} A e / d_{L}$, and $\eta=$ $E_{r} I / d_{L}$. The validity of the smeared approach is discussed in Section 4.1. 


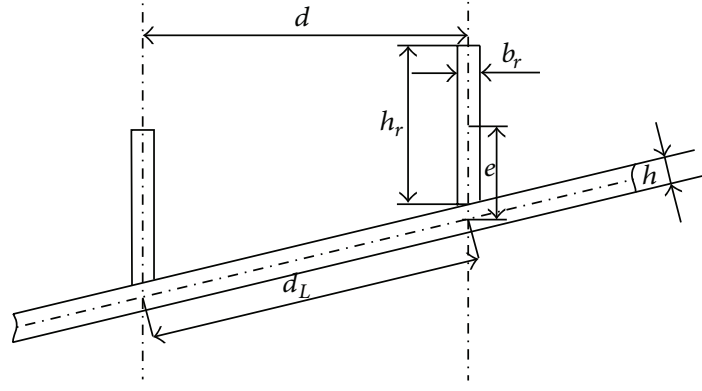

Figure 2: The parameters of the stiffeners.

When the fluid loading on the surface of the stiffened conical shell is considered, the equations of motion can be expressed as

$$
\begin{gathered}
L_{11} u_{c}+L_{12} v_{c}+L_{13} w_{c}-\rho \bar{h} \frac{\partial^{2} u_{c}}{\partial t^{2}}=0, \\
L_{21} u_{c}+L_{22} v_{c}+L_{23} w_{c}-\rho \bar{h} \frac{\partial^{2} v_{c}}{\partial t^{2}}=0, \\
L_{31} u_{c}+L_{32} v_{c}+L_{33} w_{c}-\rho \bar{h} \frac{\partial^{2} w_{c}}{\partial t^{2}}+p_{a}=0 .
\end{gathered}
$$

With the power series solution, the displacements of the conical shell are given by [7]

$$
\begin{aligned}
u_{c}(x, \theta, t) & =u_{c}(x) \cos (n \theta) e^{-j \omega t} \\
& =\sum_{n=0}^{\infty} \mathbf{u}_{c, n} \cdot \mathbf{x}_{n} \cos (n \theta) e^{-j \omega t}, \\
v_{c}(x, \theta, t) & =v_{c}(x) \sin (n \theta) e^{-j \omega t} \\
& =\sum_{n=0}^{\infty} \mathbf{v}_{c, n} \cdot \mathbf{x}_{n} \sin (n \theta) e^{-j \omega t}, \\
w_{c}(x, \theta, t) & =w_{c}(x) \cos (n \theta) e^{-j \omega t} \\
& =\sum_{n=0}^{\infty} \mathbf{w}_{c, n} \cdot \mathbf{x}_{n} \cos (n \theta) e^{-j \omega t},
\end{aligned}
$$

where the vectors $\mathbf{u}_{c, n}, \mathbf{v}_{c, n}$, and $\mathbf{w}_{c, n}$ are

$$
\begin{aligned}
\mathbf{u}_{c, n} & =\left[u_{c 1}(x) \cdots u_{c 8}(x)\right], \\
\mathbf{v}_{c, n} & =\left[v_{c 1}(x) \cdots v_{c 8}(x)\right], \\
\mathbf{w}_{c, n} & =\left[w_{c 1}(x) \cdots w_{c 8}(x)\right] .
\end{aligned}
$$

$\mathbf{x}_{n}$ is the vector of the eight unknown coefficients

$$
\mathbf{x}_{n}=\left[\begin{array}{llllllll}
a_{0} & a_{1} & b_{0} & b_{1} & c_{0} & c_{1} & c_{2} & c_{3}
\end{array}\right]^{T} .
$$

The fluid loading effects on the conical shell are taken into account using an approximate method by dividing the conical shell into $N$ narrow strips and each of these strips is treated as a cylindrical shell. The low frequency range is considered here, where the variation of the real wave number that is used to calculate the fluid loading parameter is approximately a linear function of $R_{0}$ [7]. A qualitative analysis on the number of cylindrical subdivisions required to represent the conical shell is given in [7]. A tolerance T\% is introduced due to the assumption that the wave number does not change along the length of the cone [7]. Thus, the length of each segment of the cone is determined by $L_{i} \leq 2 R_{0, j} T \% /(\sin \alpha)$. T\% depends on the geometry of the model and the frequency range, which is analyzed in Section 4.1. The $N$ parts are connected by 8 continuity equations at each interface.

The external pressure $p_{a}$ on every part of the conical shell is

$$
p_{a}=\frac{p_{a}^{\prime}}{\cos \alpha},
$$

where $p_{a}^{\prime}$ is the external pressure loading on the cylindrical shell, which can be approximated using an infinite model, as shown in [18], and is given by

$$
\begin{gathered}
p_{a}^{\prime}=\frac{\rho h c_{L}^{2}}{\bar{R}_{0}^{2}} F_{L} w_{c}, \\
F_{L}= \begin{cases}-\Omega^{2} \frac{\bar{R}_{0}}{h} \frac{\rho_{f}}{\rho} \frac{H_{n}\left(\beta_{f}\right)}{\beta_{f} H_{n}^{\prime}\left(\beta_{f}\right)}, \quad k_{f}>k_{n}, \\
-\Omega^{2} \frac{\bar{R}_{0}}{h} \frac{\rho_{f}}{\rho} \frac{K_{n}\left(\beta_{f}\right)}{\beta_{f} K_{n}^{\prime}\left(\beta_{f}\right)}, \quad k_{f}<k_{n},\end{cases} \\
\beta_{f}= \begin{cases}\bar{R}_{0} \sqrt{k_{f}^{2}-k_{n}^{2}}, & k_{f}>k_{n}, \\
\bar{R}_{0} \sqrt{k_{n}^{2}-k_{f}^{2}}, & k_{f}<k_{n},\end{cases}
\end{gathered}
$$

where $\Omega=\omega \bar{R}_{0} / c_{L}$ is the nondimensional ring frequency, $k_{f}=\omega / c_{f}$ is the fluid wave number, and $\rho_{f}, c_{f}$ are the density and speed of sound in the fluid, respectively. $k_{n}$ is the hull axial wave number and $\omega$ is the radian frequency. $\bar{R}_{0}$ is the mean radius of the conical shell segment. $H_{n}$ is the Hankel function of order $n$. $K_{n}$ is the modified Bessel function of order $n . H_{n}^{\prime}$ and $K_{n}^{\prime}$ are their derivatives with respect to the argument.

2.2. Boundary Conditions and Excitation. The dynamic response of the conical shell for each circumferential mode number $n$ is expressed in terms of $\mathbf{x}_{n}=$ $\left[\begin{array}{llllllll}a_{0} & a_{1} & b_{0} & b_{1} & c_{0} & c_{1} & c_{2} & c_{3}\end{array}\right]^{T}$ for each segment of the conical shell, which can be solved by continuity conditions between the segments and boundary conditions at the ends. The various boundary conditions for the conical shell are as follows [8].

Free end:

$$
\begin{gathered}
N_{x}=0, \\
N_{x \theta}+\frac{M_{x \theta}}{R_{0}}=0, \\
V_{x}=0, \\
M_{x}=0 ;
\end{gathered}
$$




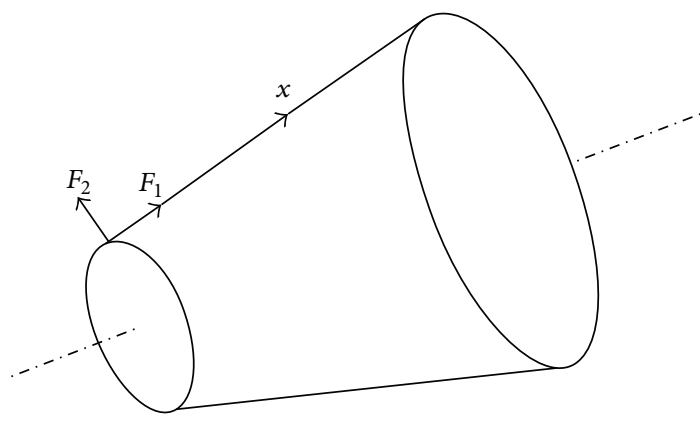

FIGURE 3: Excitation along the generator and normal to the surface at the smaller end of the conical shell.

clamped end:

$$
u_{c}=0, \quad v_{c}=0, \quad w_{c}=0, \quad \frac{\partial w_{c}}{\partial x}=0 ;
$$

shear-diaphragm (SD) end:

$$
\widetilde{N}_{x}=0, \quad v_{c}=0, \quad \widetilde{w}_{c}=0, \quad M_{x}=0,
$$

where, for the SD boundary condition, the change from the conical coordinate system defined in Figure 1 to the cylindrical coordinate system is considered. $\widetilde{N}_{x}$ and $\widetilde{w}_{c}$ are expressed as

$$
\begin{gathered}
\widetilde{N}_{x}=N_{x} \cos \alpha-V_{x} \sin \alpha, \\
\widetilde{w}_{c}=u_{c} \sin \alpha+w_{c} \cos \alpha .
\end{gathered}
$$

The forces and moments of a conical shell are given in the Appendix. The four boundary conditions at each end of the conical shell with the eight continuity equations at each junction of two conical segments can be arranged in matrix form $\mathbf{A X}=\mathbf{0}$, where $\mathbf{X}=\left[\mathbf{x}_{1}, \mathbf{x}_{2}, \ldots, \mathbf{x}_{n}\right]$.

Two excitation cases as shown in Figure 3 are considered. $F_{1}$ is the excitation along the $x$ direction. For the free end boundary condition at the end of the conical shell, the external force results in a modification of the force equilibrium at the end given by (9), which now becomes [11]

$$
N_{x}=\frac{1}{R_{1}} F_{0} \delta\left(x-x_{0}\right) \delta\left(\theta-\theta_{0}\right) e^{-j \omega t} .
$$

Excluding the harmonic time dependency, multiplying (16) by $\cos (n \theta)$, and integrating from $-\pi$ to $\pi$ yielded

$$
N_{x}=\varepsilon F_{0} \cos (n \theta),
$$

where $\varepsilon=1 / 2 \pi a$ if $n=0$ and $\varepsilon=1 / \pi a$ if $n \neq 0$. Similarly, $c$ is the excitation normal to the shell surface. In this case, (11) becomes

$$
V_{x}=\varepsilon F_{0} \cos (n \theta)
$$

Point excitation in any direction can be expressed by these two excitations. The FRF can be calculated for the case of a point force located at any junction. The axial and radial displacements are obtained from the components of the displacements,

$$
\begin{aligned}
& u_{0}=u_{c} \cos \alpha-w_{c} \sin \alpha, \\
& w_{0}=w_{c} \cos \alpha+u_{c} \sin \alpha .
\end{aligned}
$$

\section{Far-Field Sound Pressure}

In this section, the theory for the boundary element method is used to demonstrate that the far-field acoustic pressure of the shell can be solved by superposing the pressure of the individual elements. An analytical solution of the acoustic pressure radiated by a cylindrical piston in a cylindrical baffle is used to obtain the pressure of conical elements.

3.1. Acoustic Transfer Vector. According to the Helmholtz boundary integral equation, the acoustic pressure radiated by the arbitrary surface can be expressed as [19]

$$
\begin{aligned}
C(\mathbf{r}) & p(\mathbf{r}) \\
& =\int_{S_{0}}\left(p\left(\mathbf{r}_{s}\right) \frac{\partial}{\partial n} G\left(\mathbf{r}, \mathbf{r}_{s}\right)+j \rho_{f} \omega G\left(\mathbf{r}, \mathbf{r}_{s}\right) v\left(\mathbf{r}_{s}\right)\right) d S,
\end{aligned}
$$

where $\mathbf{r}_{s}$ is the position vector on the vibrating surface and $\mathbf{r}$ is the position of the observation field point. $p\left(\mathbf{r}_{s}\right)$ and $v\left(\mathbf{r}_{s}\right)$ are the surface pressure and normal velocity, respectively. $G\left(\mathbf{r}, \mathbf{r}_{s}\right)=e^{-i k_{f} R} / 4 \pi R$ is free-space Green's function and $R=$ $\left|\mathbf{r}-\mathbf{r}_{s}\right|$.

By discretizing the surface into $M$ elements, (20) can be expressed as

$$
C(\mathbf{r}) p(\mathbf{r})=\sum_{i=1}^{M} \int_{S_{i}}\left(p\left(\mathbf{r}_{s}\right) \frac{\partial}{\partial n} G\left(\mathbf{r}, \mathbf{r}_{s}\right)+j \rho \omega G\left(\mathbf{r}, \mathbf{r}_{s}\right) v\right) d S .
$$

Equation (21) can be rearranged as [15]

$$
p(\mathbf{r})=[\mathbf{C}]_{M \times 1}^{T}\left[\mathbf{p}_{s}\right]+[\mathbf{D}]_{M \times 1}^{T}[\mathbf{v}],
$$

where $\mathbf{C}$ and $\mathbf{D}$ are the vectors independent of the surface velocity. The relationship between the surface pressure vector $\mathbf{p}_{s}$ and the normal velocity $\mathbf{v}$ can be deduced as

$$
[A]_{M \times M}\left\{\mathbf{p}_{s}\right\}=[B]_{M \times M}\{\mathbf{v}\},
$$

where the matrices $A, B$ are independent of the surface velocity but depend on the shape of the structure and frequency. Equation (22) can be expressed as

$$
\begin{aligned}
p(\mathbf{r}) & =\left([\mathbf{C}]_{M \times 1}^{T}[A]_{M \times M}^{-1}[B]_{M \times M}+[\mathbf{D}]_{M \times 1}^{T}\right)[\mathbf{v}] \\
& =[\mathbf{G}]_{M \times 1}^{T}[\mathbf{v}] .
\end{aligned}
$$

The vector $\mathbf{G}$ is called Acoustic Transfer Vector (ATV), which is an array of transfer functions between the surface normal velocities and the pressure at the field point. The ATV depends on frequency, geometry, acoustical parameters of the fluid, and position of the observation point. 


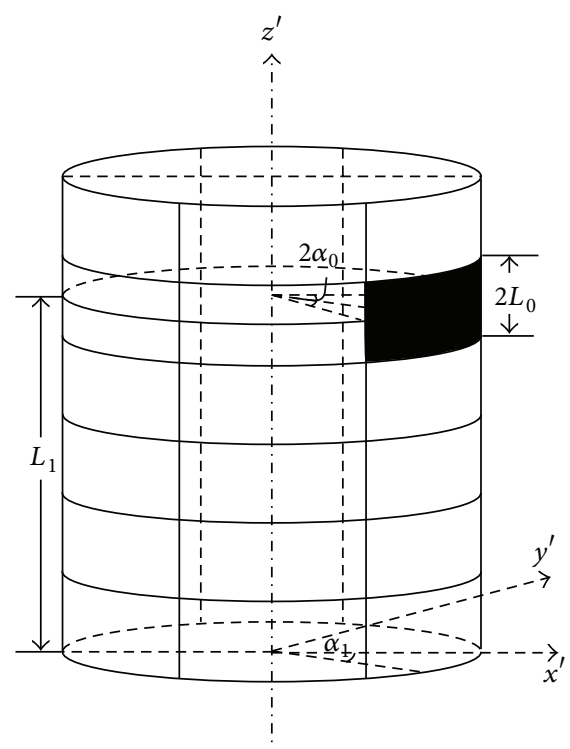

(a)

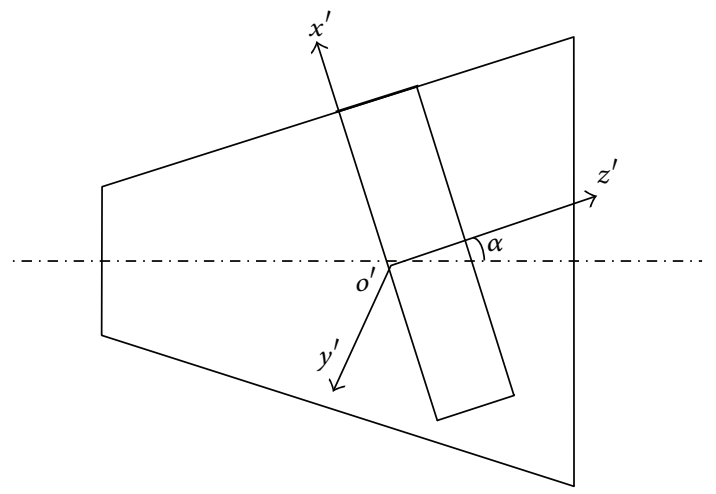

(b)

FIgURE 4: The cylindrical baffle and piston.

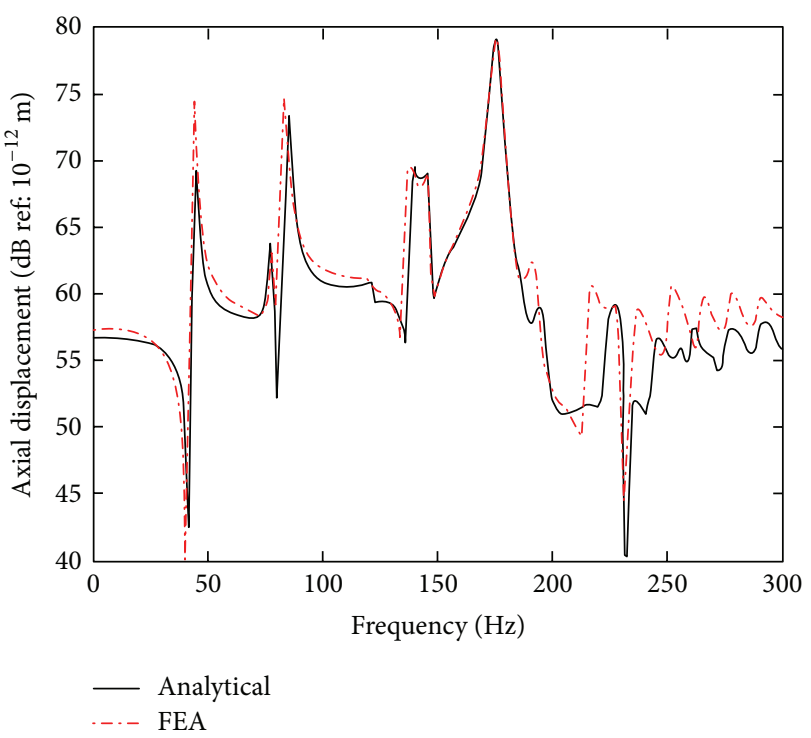

FIGURE 5: Range of validity for the stiffeners smeared over the conical shell.

3.2. Element Radiation Superposition Method. The conical shell is divided into $M$ elements, $[\mathbf{G}]_{M \times 1}^{T}=\left[g_{1}, g_{2}, \ldots, g_{M}\right]$. Each element of the ATV, $g_{i}$, is equal to the acoustic pressure radiated by the corresponding surface element vibrating with unity surface velocity while the other elements are at rest. For the case where only one element vibrates with the velocity $v_{i}$, the radiated pressure of the field point at $\mathbf{r}$ is given by

$$
p_{i}(\mathbf{r})=\left[g_{1}, g_{2}, \ldots, g_{M}\right]\left[0,0, \ldots, v_{i}, \ldots, 0\right]^{T}
$$

Therefore, given the velocities $\left[v_{1}, v_{2}, \ldots, v_{i}, \ldots, v_{M}\right]^{T}$, the radiated pressure is

$$
p(\mathbf{r})=p_{1}(\mathbf{r})+p_{2}(\mathbf{r})+\cdots+p_{M}(\mathbf{r}) .
$$

Hence, the total radiation pressure is the superposition of the acoustic pressures radiated by the surface elements vibrating with a rigid baffle. Since the velocities $\left[v_{1}, v_{2}, \ldots, v_{i}, \ldots, v_{M}\right]$ can be obtained from the solution of dynamic response of the conical shell, the main task is to find the acoustic transfer values $\left[g_{1}, g_{2}, \ldots, g_{M}\right]$.

According to Wang [15], the ATV of the element on a cylindrical shell is equal to the acoustic pressure radiated by a baffled piston vibrating with unity velocity. The farfield acoustic pressure radiated by a cylindrical piston in a cylindrical baffle can be obtained by solving the Helmholtz differential equation in closed form, which is given by [20]

$$
\begin{aligned}
p(R, \theta, \phi)= & \frac{2 \rho c \alpha_{0} L_{0}}{\pi^{2} R \sin \theta} j_{0}\left(k L_{0} \cos \theta\right) e^{j k\left(R-L_{1} \cos \theta\right)} \\
& \times \sum_{n=-\infty}^{\infty} \frac{(-j)^{n} j_{0}\left(n \alpha_{0}\right)}{H_{n}^{(1)^{\prime}}(k a \sin \theta)} e^{j n\left(\phi-\alpha_{1}\right)} \cdot v,
\end{aligned}
$$

where $2 L_{0}$ is the axial length of the piston, $2 \alpha_{0}$ is the radial angle of the piston, $L_{1}$ is the axial distance between the center of the piston and the origin, and $\alpha_{1}$ is the azimuth angle of the center of the piston. All of these parameters are shown in Figure 4(a). $v$ is the velocity of the baffled piston. For a velocity equal to unity, the ATV of the each cylindrical shell element $g_{i}$ can be obtained from $g_{i}=p_{i}(R, \theta, \phi)$.

The radiated pressure from each element of the conical shell can be approximated by the analytical solution of the radiated pressure of a piston in a cylindrical baffle, as shown 


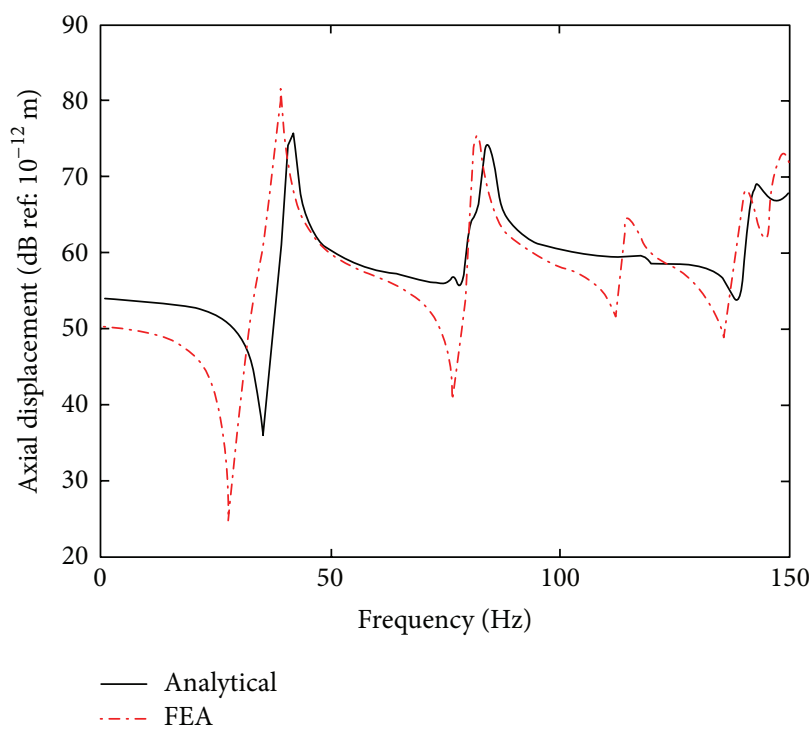

(a)

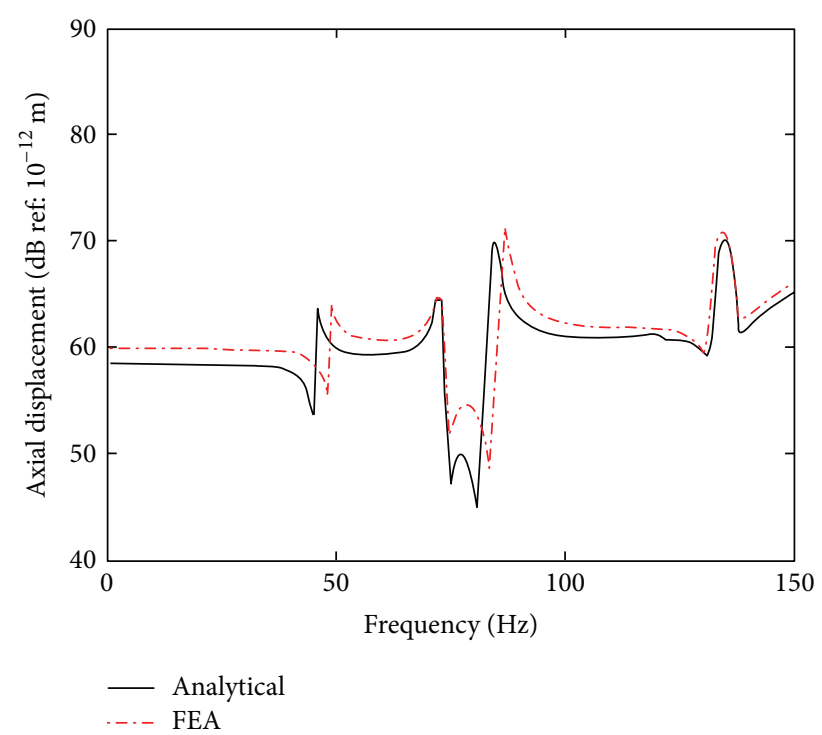

(b)

Figure 6: Comparison with FEA results when the space or size of stiffeners changes. (a) Results when the space between stiffeners $d=0.42$; (b) results when cross-section of the stiffeners is $0.028 \mathrm{~m} \times 0.04 \mathrm{~m}$.

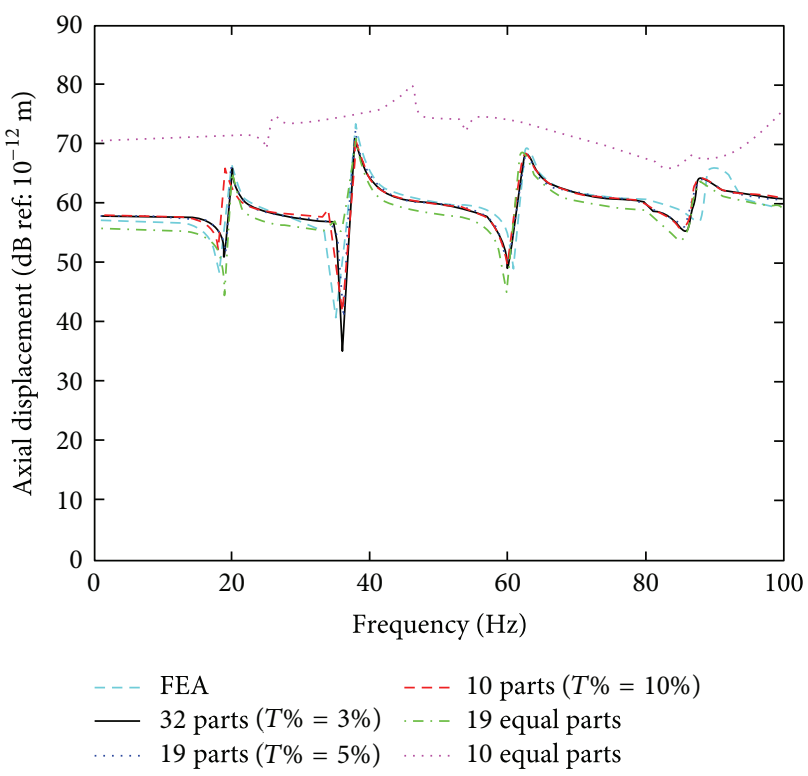

FIGURE 7: Comparison for different divisions for the conical shell.

in Figure 4(b). The radius of this cylindrical baffle is equal to the mean radius of the conical strip which includes this element. The ATV of the piston in the cylindrical baffle is solved using its local coordinate. The angle between the axis of the conical shell and the axis of the cylindrical baffle is the semivertex angle of the conical shell. In this way, the ATV elements of the conical shell $\left[g_{1}, g_{2}, \ldots, g_{M}\right]$ can be obtained and the total radiated pressure of the conical shell is solved by summing up the products of the individual transfer quantities and the corresponding velocities.

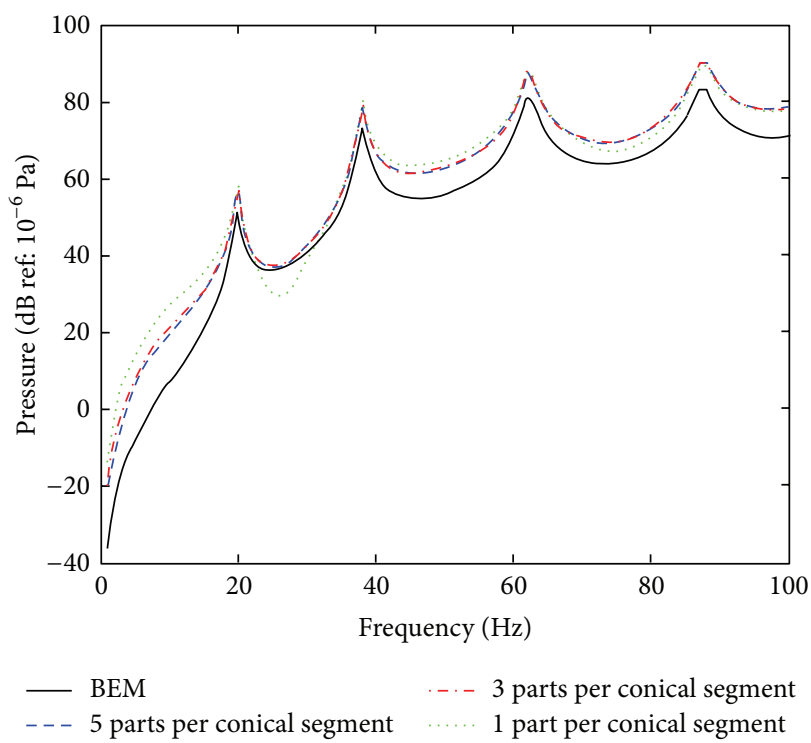

FIGURE 8: Validity and convergence of solving far-field pressure.

\section{Results for Structural and Acoustic Responses for a Conical Shell}

The structural and acoustic responses for the conical shell under harmonic excitation are presented using the following parameters: $L=8.9 \mathrm{~m}, R_{1}=0.5 \mathrm{~m}, R_{2}=3.25 \mathrm{~m}$, and $h=0.014 \mathrm{~m}$. The stiffeners have a rectangular cross-section of $0.014 \mathrm{~m} \times 0.04 \mathrm{~m}\left(b_{r}=0.014 \mathrm{~m}, h_{r}=0.04 \mathrm{~m}\right)$ and are evenly spaced by $d=0.21 \mathrm{~m}$. The material properties for steel are density $\rho=7800 \mathrm{kgm}^{-3}$, Young's modulus $E=2.1 \times$ $10^{11} \mathrm{Nm}^{-2}$, and Poisson's ratio $\mu=0.3$. Structural damping 


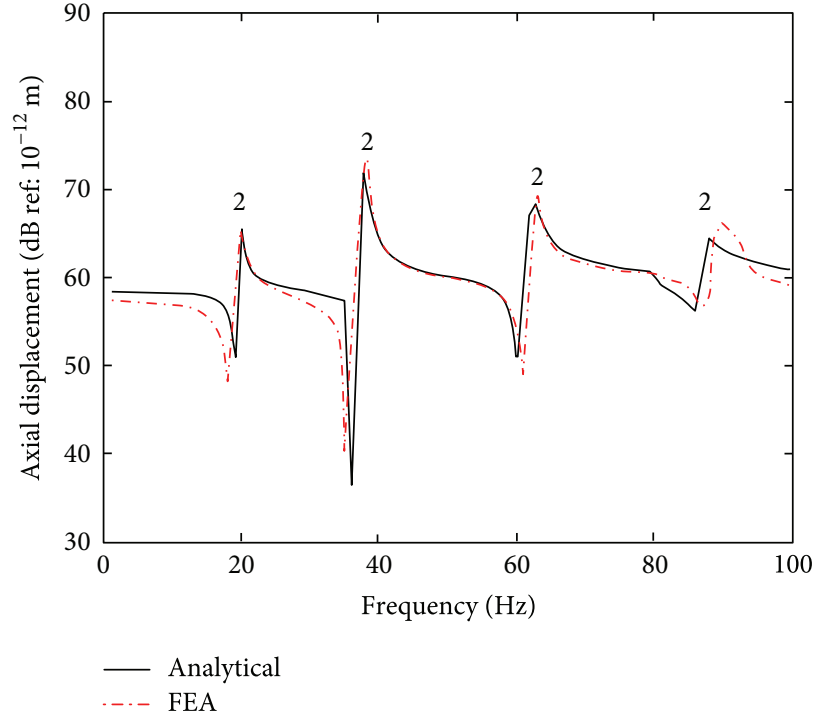

(a)

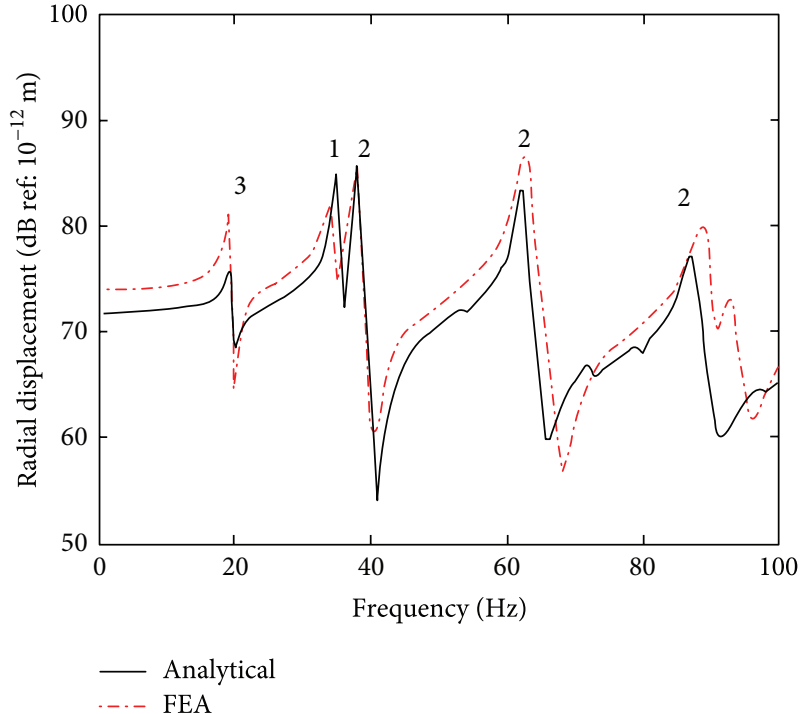

(b)

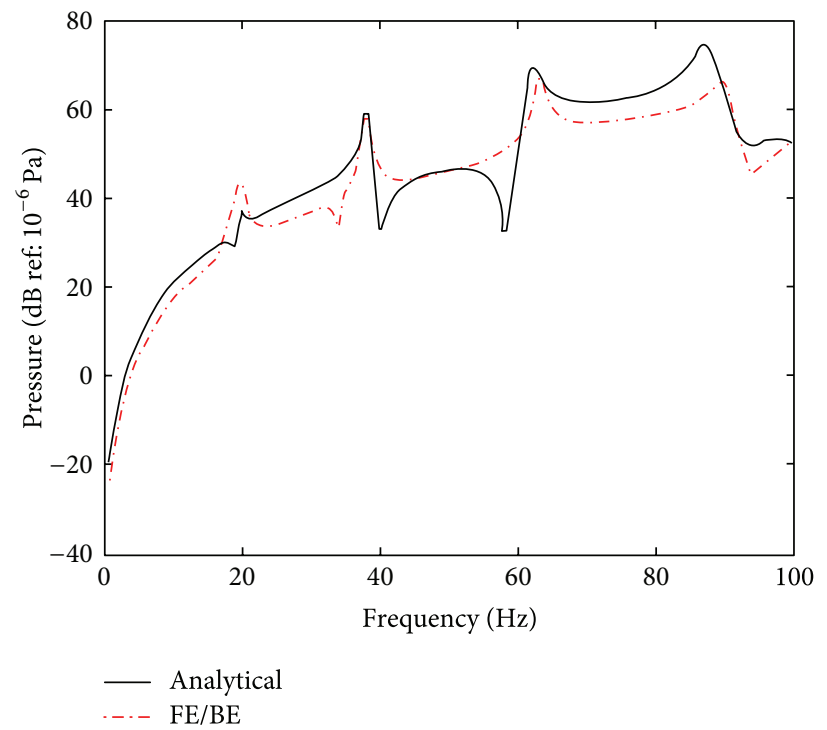

(c)

FIgURE 9: Structural and acoustic responses due to $F_{1}$ (a) axial displacement, (b) radial displacement, and (c) far-field pressure.

is introduced using complex Young's modulus $E\left(1-j \eta_{s}\right)$, where $\eta_{s}=0.02$ is the structural loss factor. The surrounding fluid has a density of $\rho_{f}=1000 \mathrm{kgm}^{-3}$ and speed of sound $c_{f}=1500 \mathrm{~ms}^{-1}$. The structural responses are predicted at a point equal to the drive point of the excitation, as shown in Figure 3. The distance between the far-field point and the axis of the conical shell is $100 \mathrm{~m}$. The circumferential angle of the observation point is $\pi / 2$ and its axial position is equal to the axial position of the drive point. The boundary conditions considered here are free at the smaller end of the conical shell and clamped at the larger end.

A finite element model is developed in ANSYS to validate the results obtained from analytical model presented here. The conical shell model and the ring stiffeners are built using Shell 63 elements and Beam 188 elements, respectively. Fluid
30 and Fluid 130 elements are used to simulate the external fluid and the absorbing boundaries around the fluid domain. ANSYS is used to obtain the structural responses. SYSNOISE is then used to calculate the far-field pressure using the direct boundary element method.

4.1. Validity and Convergence. The validity of smeared approach for the stiffened conical shell and the convergence of dividing the conical shell are discussed in what follows. A model of the stiffened conical shell in vacuo is built to validate the smeared approach. It is observed in Figure 5 that the analytical results for the structural responses due to $F_{1}$ match the finite element analysis (FEA) results in the low frequency range. However, the accuracy decreases rapidly when the frequency is above $180 \mathrm{~Hz}$, where the structural wavelength 


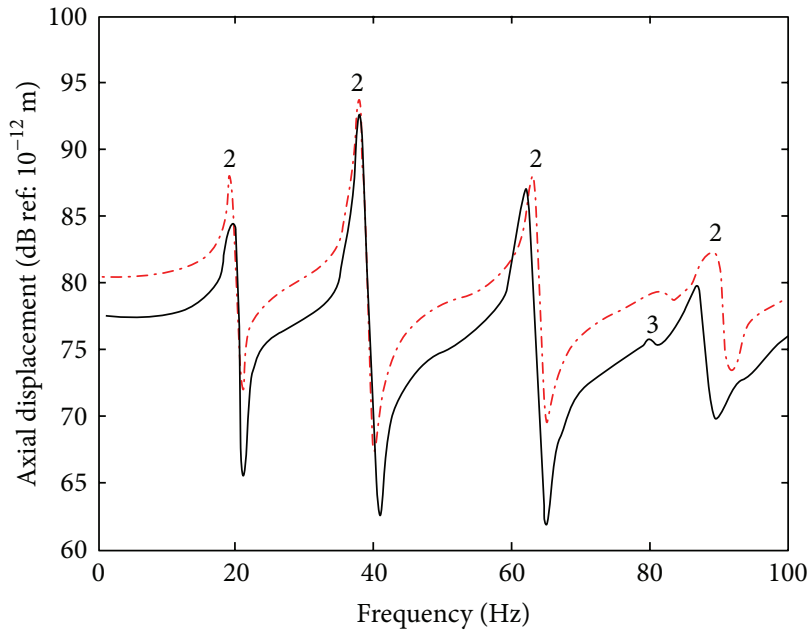

Analytical

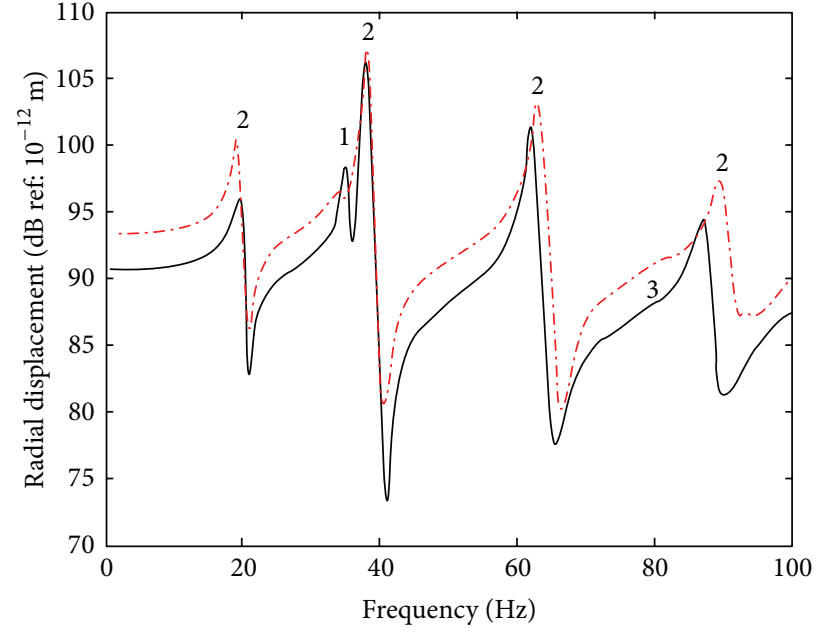

- Analytical -..- FEA

(a)

(b)

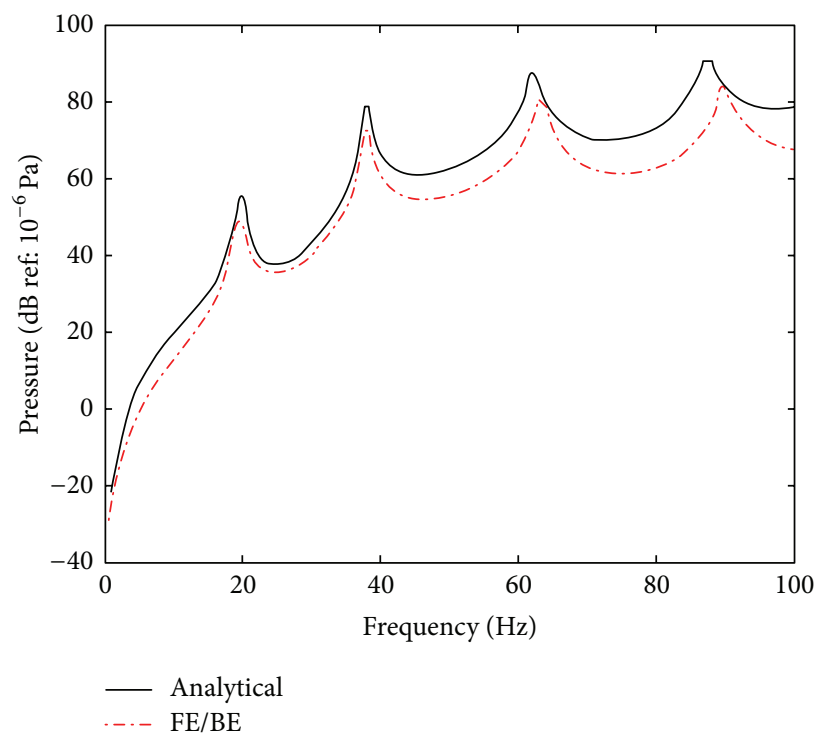

(c)

FIGURE 10: Structural and acoustic responses due to $F_{2}$ (a) axial displacement, (b) radial displacement, and (c) far-field pressure.

is of the same order as the distance between the stiffeners. It is further observed that the increase of the spacing and width of the stiffeners affects the accuracy of this method, as shown in Figures 6(a) and 6(b), respectively. To conclude, the smeared approach is only suitable when the conical shells are reinforced by closely spaced stiffeners, that is, $d / L$ is small, and sufficiently small stiffeners, that is, $b_{r} / L$ is small, in the low frequency range, where the wavelength is larger than the stiffener spacing.

As discussed in Section 2, the conical shell is divided into $N$ segments and the length of each segment is $L_{i} \leq$ $2 R_{0, i} T \% /(\sin \alpha)$. For the model in this paper, $T \%=5 \%$. The result for the structural responses due to $F_{1}$ for $T \%=5 \%$ is compared with the results for $T \%=3 \%$ and $T \%=10 \%$ in Figure 7. It can be seen that the result converged for $T=5 \%$.
Two additional cases of dividing the conical shell into parts with equal lengths are also considered in Figure 7. It shows that segments with fixed $L_{i} / R_{0, i}$ yield faster convergence than fixed length segments. The FEA result is also presented in Figure 7 to validate the converged analytical result.

The validity and convergence of the Element Radiation Superposition Method (ERSM) for solving the far-field acoustic pressure of a conical shell are discussed in what follows. Each conical segment with the length of $L_{i} \leq$ $2 R_{0, i} T \% /(\sin \alpha)$ is divided into 1,3 , and 5 parts in its axial direction, corresponding to 19,37 , and 95 parts, respectively, for $T=5 \%$ and 19 conical segments. The total number of elements for the conical shell is 1140,2220 , and 5700 , respectively, for a circumferential angle of $6^{\circ}$ for each element. Each element can be regarded as a piston in a cylindrical baffle. 


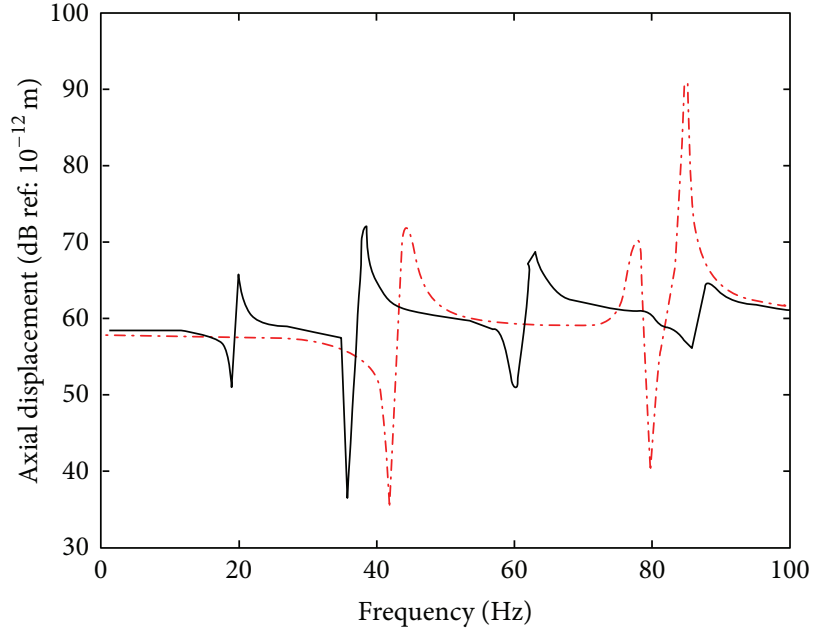

(a)

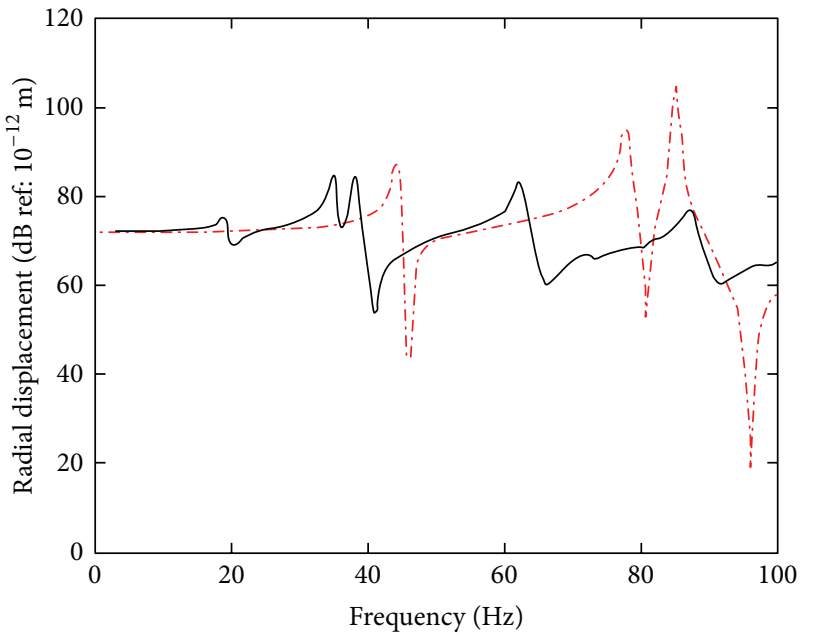

(b)

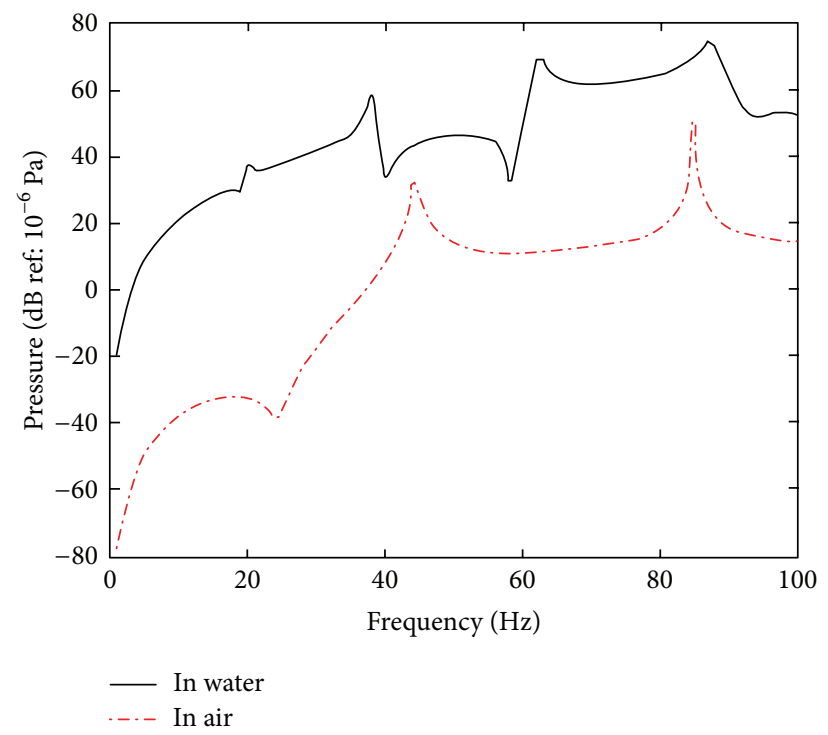

(c)

Figure 11: Comparison of conical shells in water and in air, under $F_{1}(\mathrm{a})$ axial displacement, (b) radial displacement, and (c) far-field pressure.

The results in Figure 8 show that the acoustic pressure due to $F_{2}$ converges when there are 3 parts per conical segment. The BEM result is also shown in Figure 8. As discussed in Section 3, the pressure is obtained from the velocity and ATV. In this part only, for both methods, the velocities are analytical solutions of the structural responses. The difference between ERSM and BEM lies in the solution of the ATV. The ATV for ERSM is obtained from the analytical solution of the Helmholtz differential equation for a piston baffled in a cylindrical shell, which is used to approximate a piston baffled in a conical shell, while the ATV for BEM is obtained by the numerical solution of the Helmholtz boundary integral formulation. The results in Figure 8 indicate that the locations of the peaks are well matched but the errors of amplitudes of the peaks are less than 5\% at lower frequencies and less than $10 \%$ at higher frequencies. However, ERSM has a much quicker solution than BEM, due to its avoidance of solving the Helmholtz boundary integral formulation. Therefore, ERSM is an effective method to solve the far-field pressure of conical shell.

4.2. Effect of Excitation on a Conical Shell. The structural and acoustic responses of the conical shell due to the excitation shown in Figure 3 are presented in Figures 9 and 10. The comparison between the analytical results and the FEA results indicates that the axial and radial displacements using these two methods agree well, except for some errors at higher frequencies. These errors arise due to approximate solutions for the stiffeners and the external fluid loading. The stiffeners are considered by averaging their properties over the surface of the conical shell. The fluid loading is taken into account by dividing the conical shell into narrow strips, which are treated as cylindrical shells and the external fluid loading is approximated using an infinite model. The far-field acoustic pressures obtained using the analytical and FE/BE methods are also in good agreement. Errors are caused by 


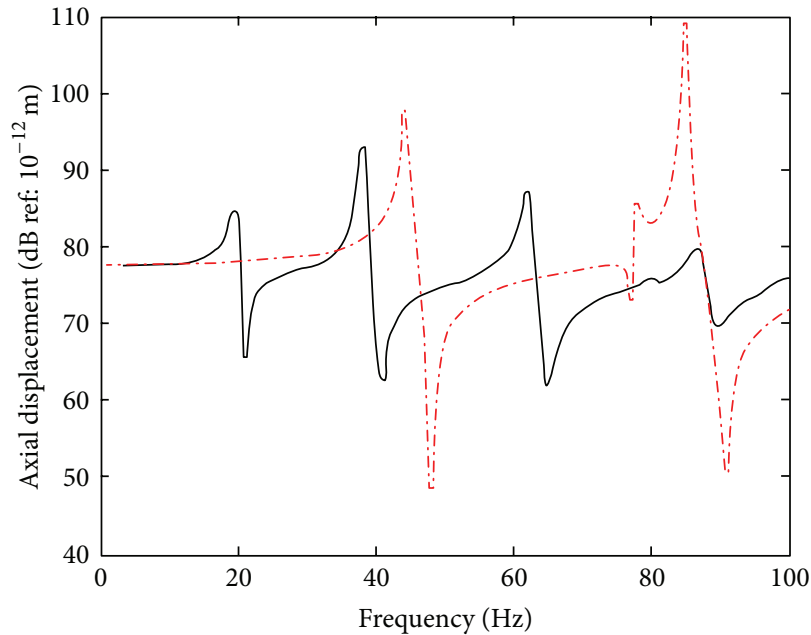

(a)

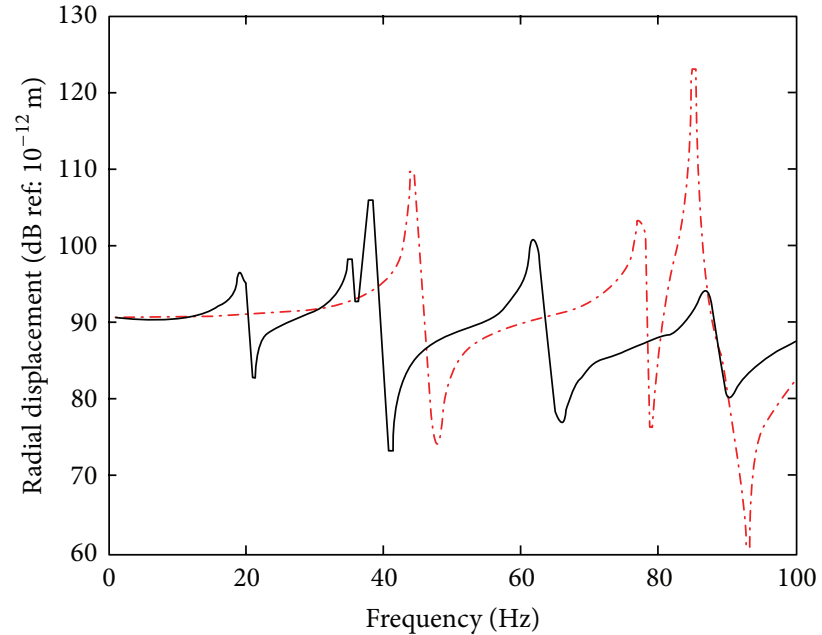

(b)

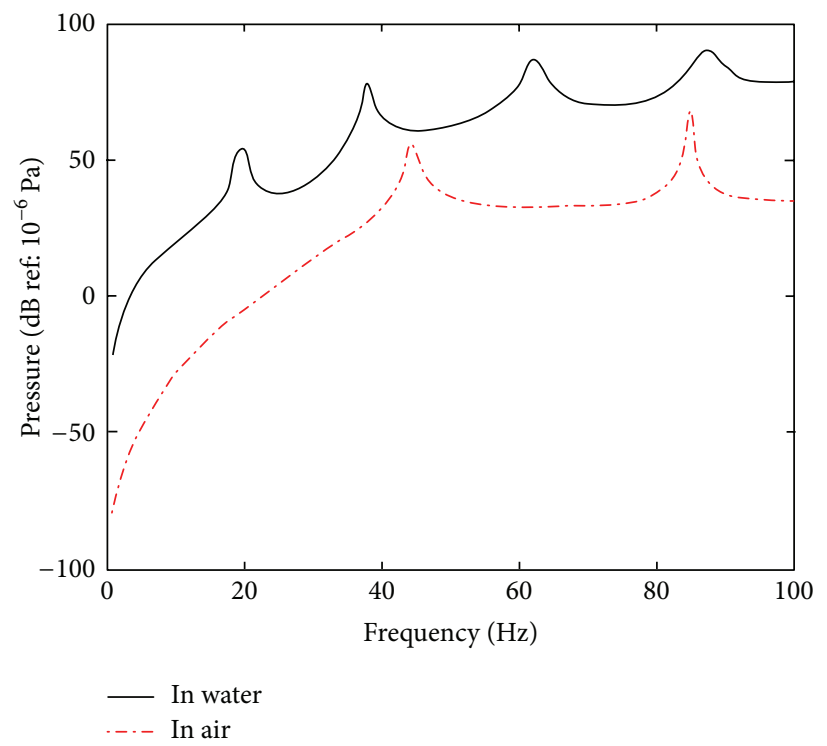

(c)

FIGURE 12: Comparison of conical shells in water and in air, under $F_{2}$ (a) axial displacement, (b) radial displacement, and (c) far-field pressure.

the velocities, which are gained from the results of structural responses, and the ERSM, as it has a different solution of the ATV from the BEM. Thus, it can be found that some errors exist, especially at higher frequencies, due to complexity and approximations of the pressure solution from ERSM. But they are acceptable and applicable in the preliminary research, in the case that the analytical methods are able to save much time of the calculations.

The contributions of the independent circumferential modes with mode order $n$ are marked in the figures of the axial and radial displacements. It is observed that, in both cases, the $n=2$ modes are predominantly excited, followed by $n=1$ and $n=3$ modes. Higher order circumferential modes do not significantly contribute to the structural responses. It is also found that both structural and acoustic responses due to the $F_{2}$ excitation are significantly higher than those due to the $F_{1}$ excitation.
4.3. Effect of External Fluid Loading. The effect of the fluid loading on the structural and acoustic responses for the two excitation cases is presented for the conical shell in air in Figures 11 and 12. It is observed that the effects of the external fluid for the two cases are similar. The fluid loading reduces the peaks of the axial and radial resonant frequencies, due to damping. Moreover, the resonant peaks shift to lower frequencies because of the mass-like effect of the fluid loading. The amplitude of the far-field pressure for the conical shell in water is significantly higher than the pressure for the conical shell in air, which indicates that the sound is transmitted more efficiently in water than in air.

4.4. Effect of Ring Stiffeners. The effect of the ring stiffeners on the structural and acoustic responses of the conical shell responses is presented in Figures 13 and 14. The ring stiffeners are observed to have a stiffening effect, resulting in an increase 


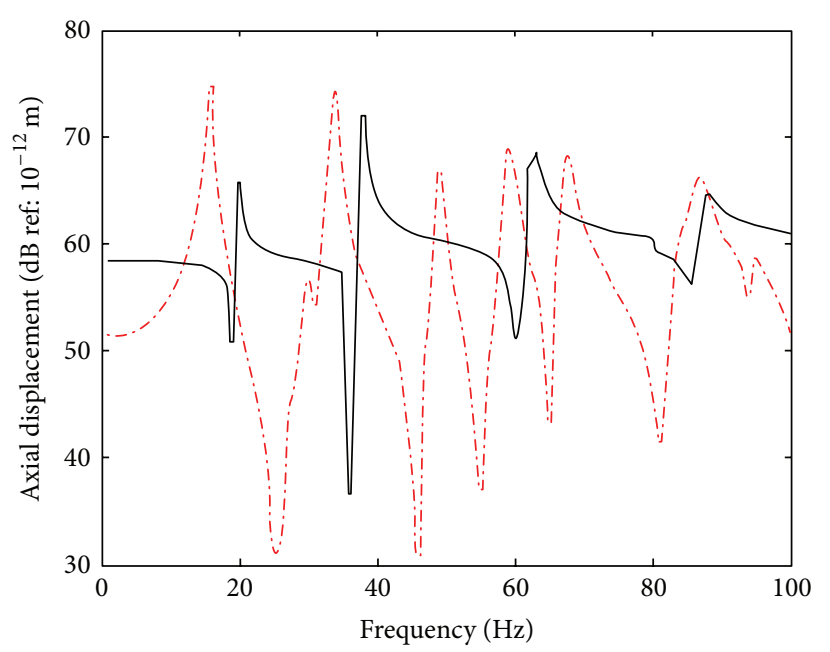

(a)

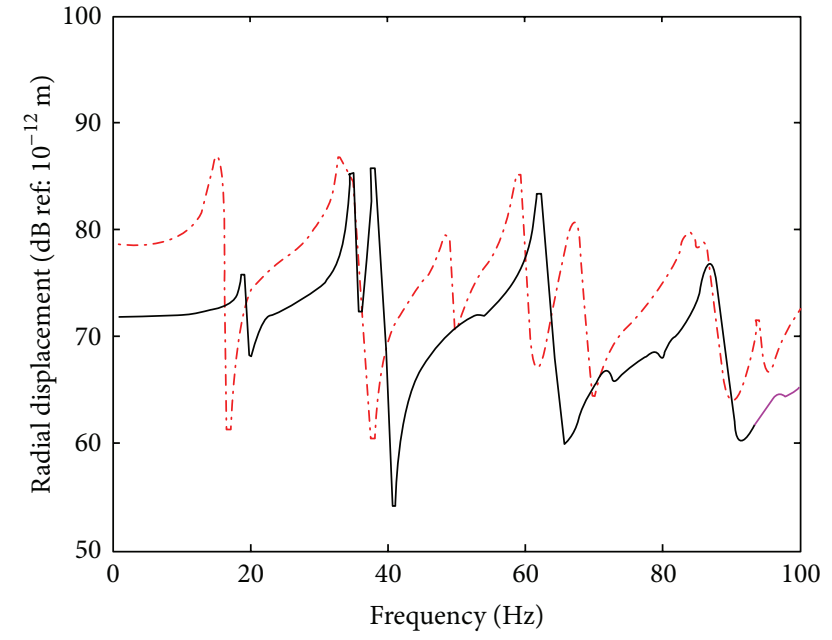

(b)

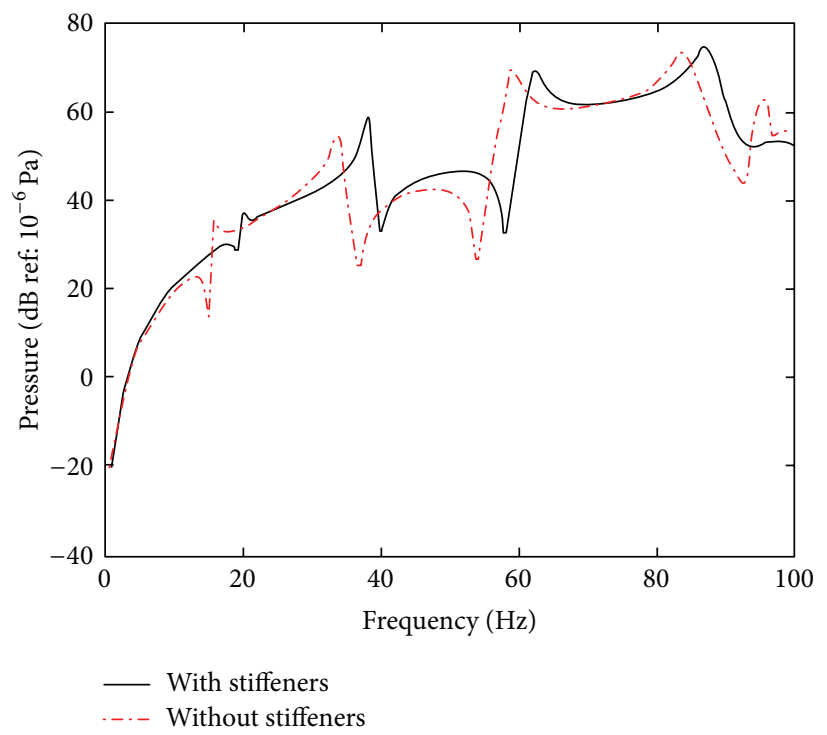

(c)

FIGURE 13: Comparison of conical shells with and without stiffeners, under $F_{1}$ (a) axial displacement, (b) radial displacement, and (c) far-field pressure.

of the resonant frequencies for both excitation cases. The shift of the resonant peaks of the far-field pressure is not as significant as that for the displacements, which shows that the added stiffness has little effect on the radiated pressure. It is further observed that the amplitudes of the pressure from conical shells with and without ring stiffeners are similar, which means that the ring stiffeners do not significantly affect the transmission of sound into the acoustic far-field.

\section{Conclusion}

An analytical method to predict the structural and acoustic responses of a stiffened conical shell immersed in fluid has been presented. The solution for the dynamic response of a conical shell was presented in the form of a power series. Fluid loading on the shell was taken into account by dividing the shell into narrow strips and stiffeners were modeled using a smeared approach. The Element Radiation Superposition Method was used to solve the far-field pressure of a conical shell. Good agreement between the analytical and FEM/BEM results was observed. Two cases of excitation by considering forces acting in different directions at the smaller end of the conical shell were examined, in which the forces acted along the generator and normal to the surface of the shell. In both cases, $n=2$ modes were predominantly excited, followed by $n=1$ and $n=3$ modes. Both the structural and acoustic responses of the conical shell under excitation in normal direction were greater than for excitation along the generator. The effects of fluid loading and ring stiffeners were examined. The results showed that external fluid loading has both damping and mass-like effects while stiffeners mainly increase the stiffness of the conical shell. 


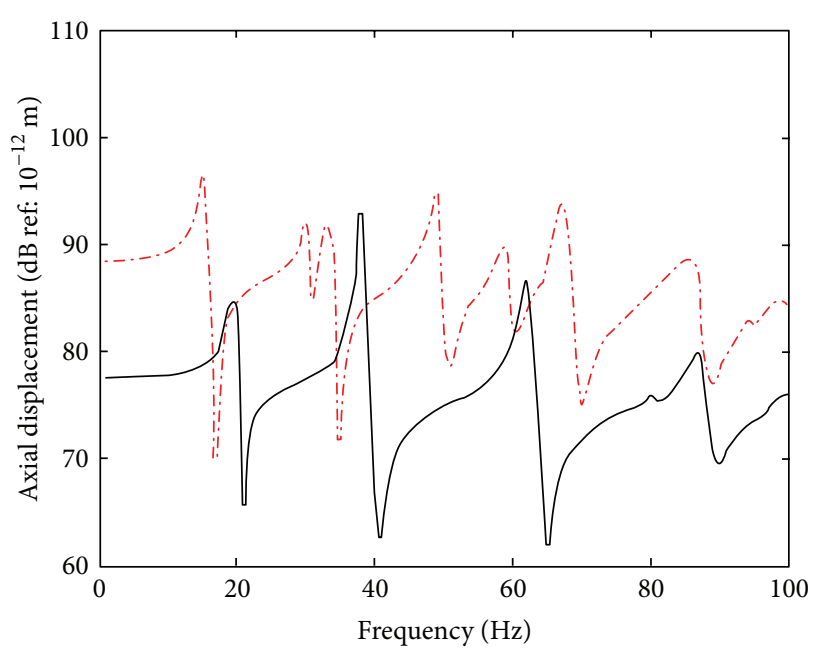

(a)

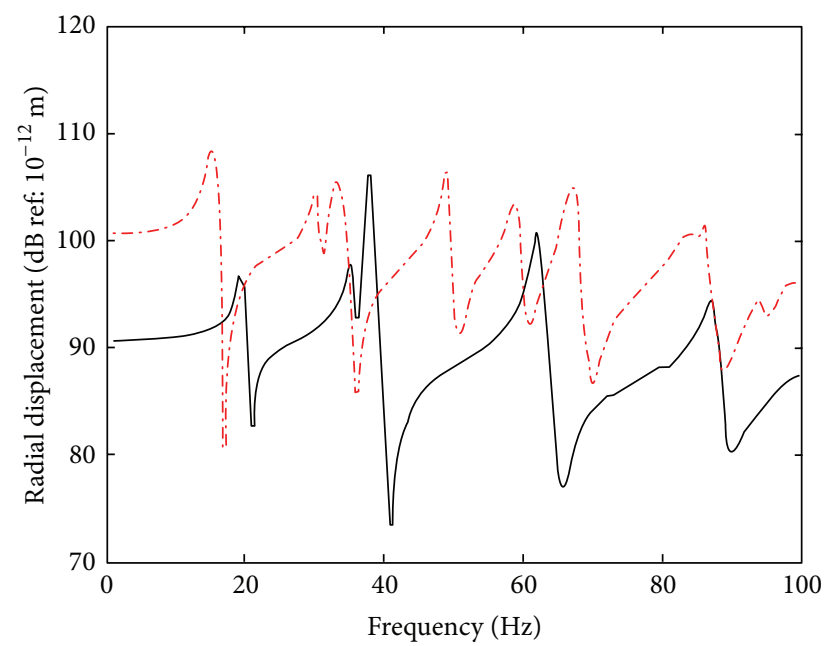

(b)

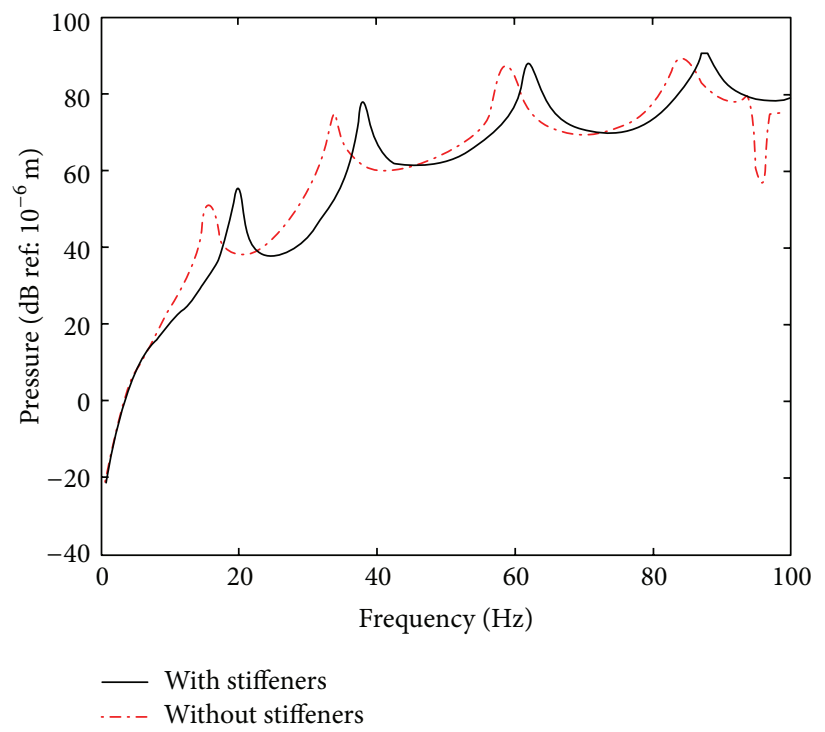

(c)

Figure 14: Comparison of conical shells with and without stiffeners, under $F_{2}$ (a) axial displacement, (b) radial displacement, and (c) far-field pressure.

\section{Appendix}

The forces and moments for a conical shell are given by [1]

$$
\begin{gathered}
N_{x}=\frac{E h}{1-\mu^{2}}\left(\frac{\partial u_{c}}{\partial x}+\mu\left(\frac{1}{R(x)} \frac{\partial v_{c}}{\partial \theta}+\frac{\sin \alpha}{R(x)} u_{c}+\frac{\cos \alpha}{R(x)} w_{c}\right)\right. \\
\left.-\frac{h^{2}}{12} \frac{\cos \alpha}{R(x)} \frac{\partial^{2} w_{c}}{\partial x^{2}}\right),
\end{gathered}
$$$$
N_{x \theta}=\frac{E h}{2(1+\mu)}\left[\frac{1}{R(x)} \frac{\partial u_{c}}{\partial \theta}+\frac{\partial v_{c}}{\partial x}-\frac{\sin \alpha}{R(x)} v_{c}+\frac{h^{2}}{12}\right.
$$$$
\times\left(-\frac{\cos \alpha}{R^{2}(x)} \frac{\partial^{2} w_{c}}{\partial \theta \partial x}+\frac{\cos ^{2} \alpha}{R^{2}(x)} \frac{\partial v_{c}}{\partial x}\right.
$$$$
\left.\left.+\frac{\cos \alpha \sin \alpha}{R^{3}(x)} \frac{\partial w_{c}}{\partial \theta}-\frac{\cos ^{2} \alpha \sin \alpha}{R^{3}} v_{c}\right)\right],
$$$$
N_{\theta x}=\frac{E h}{2(1+\mu)}\left[\frac{1}{R(x)} \frac{\partial u_{c}}{\partial \theta}+\frac{\partial v_{c}}{\partial x}-\frac{\sin \alpha}{R(x)} v_{c}\right.
$$

$$
\left.\left.+\frac{\sin \alpha \cos ^{2} \alpha}{R^{3}(x)} u_{c}+\frac{\cos ^{3} \alpha}{R^{3}(x)} w_{c}\right)\right]
$$

$$
\begin{array}{r}
N_{\theta}=\frac{E h}{1-\mu^{2}}\left[\frac{1}{R(x)} \frac{\partial v_{c}}{\partial \theta}+\mu \frac{\partial u_{c}}{\partial x}+\frac{\sin \alpha}{R(x)} u_{c}+\frac{\cos \alpha}{R(x)} w_{c}\right. \\
+\frac{h^{2}}{12}\left(\frac{\cos \alpha}{R^{3}(x)} \frac{\partial^{2} w_{c}}{\partial \theta^{2}}+\frac{\sin \alpha \cos \alpha}{R^{2}(x)} \frac{\partial w_{c}}{\partial x}\right.
\end{array}
$$$$
+\frac{h^{2}}{12}\left(\frac{\cos \alpha}{R^{2}(x)} \frac{\partial^{2} w_{c}}{\partial \theta \partial x}-\frac{\cos \alpha \sin \alpha}{R^{3}(x)} \frac{\partial w_{c}}{\partial \theta}\right.
$$ 


$$
\begin{aligned}
& \left.\left.+\frac{\cos ^{2} \alpha}{R^{3}(x)} \frac{\partial u_{c}}{\partial \theta}+\frac{\cos ^{2} \alpha \sin \alpha}{R^{3}(x)} v_{c}\right)\right], \\
& M_{x}=\frac{E h^{3}}{12\left(1-\mu^{2}\right)} \\
& \times\left[-\frac{\partial^{2} w_{c}}{\partial x^{2}}+\mu\right. \\
& \times\left(\frac{\cos \alpha}{R^{2}(x)} \frac{\partial v_{c}}{\partial \theta}-\frac{1}{R^{2}(x)} \frac{\partial^{2} w_{c}}{\partial \theta^{2}}-\frac{\sin \alpha}{R(x)} \frac{\partial w_{c}}{\partial x}\right) \\
& \left.+\frac{\cos \alpha}{R(x)} \frac{\partial u_{c}}{\partial x}\right], \\
& M_{\theta}=\frac{E h^{3}}{12\left(1-\mu^{2}\right)} \\
& \times\left[-\frac{1}{R^{2}(x)} \frac{\partial^{2} w_{c}}{\partial \theta^{2}}-\frac{\sin \alpha}{R(x)} \frac{\partial w_{c}}{\partial x}-\mu \frac{\partial^{2} w_{c}}{\partial x^{2}}\right. \\
& \left.-\frac{\cos \alpha \sin \alpha}{R^{2}(x)} u_{c}-\frac{\cos ^{2} \alpha}{R^{2}(x)} w_{c}\right], \\
& M_{x \theta}=\frac{E h^{3}}{12(1+\mu)} \\
& \times\left(-\frac{1}{R(x)} \frac{\partial^{2} w_{c}}{\partial x \partial \theta}+\frac{\cos \alpha}{R(x)} \frac{\partial v_{c}}{\partial x}+\frac{\sin \alpha}{R^{2}(x)} \frac{\partial w_{c}}{\partial \theta}\right. \\
& \left.-\frac{\sin \alpha \cos \alpha}{R^{2}} v_{c}\right) \\
& M_{\theta x}=\frac{E h^{3}}{24(1+\mu)} \\
& \times\left(-\frac{2}{R(x)} \frac{\partial^{2} w_{c}}{\partial x \partial \theta}+\frac{2 \sin \alpha}{R^{2}(x)} \frac{\partial w_{c}}{\partial \theta}+\frac{\cos \alpha}{R(x)} \frac{\partial v_{c}}{\partial x}\right. \\
& \left.-\frac{\cos \alpha}{R^{2}(x)} \frac{\partial u_{c}}{\partial \theta}-\frac{\cos \alpha \sin \alpha}{R^{2}(x)} v_{c}\right), \\
& Q_{x}=\frac{1}{R(x)} \frac{\partial\left(R(x) M_{x}\right)}{\partial x}+\frac{1}{R(x)} \frac{\partial\left(M_{\theta x}\right)}{\partial \theta}-\frac{\sin \alpha}{R(x)} M_{\theta}, \\
& Q_{\theta}=\frac{1}{R(x)} \frac{\partial\left(M_{\theta}\right)}{\partial \theta}+\frac{1}{R(x)} \frac{\partial\left(R M_{x \theta}\right)}{\partial x}+\frac{\sin \alpha}{R(x)} M_{\theta x}, \\
& V_{x}=Q_{x}+\frac{1}{R} \frac{\partial M_{x \theta}}{\partial \theta},
\end{aligned}
$$

where $E, \mu$, and $h$, respectively, are Young's modulus, Poisson's ratio, and shell thickness.

\section{Conflict of Interests}

The authors declare that there is no conflict of interests regarding the publication of this paper.

\section{Acknowledgments}

All the work in this paper obtains great support from the National Natural Science Foundation of China (51179071) and the Fundamental Research Funds for the Central Universities, HUST: 2012QN056.

\section{References}

[1] A. W. Leissa, Vibration of Shells, American Institue of Physics, New York, NY, USA, 1993.

[2] H. Saunders, E. J. Wisniewski, and P. R. Paslay, "Vibrations of conical shells," Journal of the Acoustical Society of America, vol. 32, pp. 765-772, 1960.

[3] H. Garnet and J. Kempner, "Axisymmetric free vibration of conical shells," Journal of Applied Mechanics, vol. 31, pp. 458466, 1964.

[4] L. Tong, "Free vibration of orthotropic conical shells," International Journal of Engineering Science, vol. 31, no. 5, pp. 719-733, 1993.

[5] Y. P. Guo, "Normal mode propagation on conical shells," Journal of the Acoustical Society of America, vol. 96, no. 1, pp. 256-264, 1994.

[6] Y. P. Guo, "Fluid-loading effects on waves on conical shells," Journal of the Acoustical Society of America, vol. 97, no. 2, pp. 1061-1066, 1995.

[7] M. Caresta and N. J. Kessissoglou, "Vibration of fluid loaded conical shells," Journal of the Acoustical Society of America, vol. 124, no. 4, pp. 2068-2077, 2008.

[8] M. Caresta and N. J. Kessissoglou, "Free vibrational characteristics of isotropic coupled cylindrical-conical shells," Journal of Sound and Vibration, vol. 329, no. 6, pp. 733-751, 2010.

[9] C. C. Chien, H. Rajiyah, and S. N. Atluri, "An effective method for solving the hypersingular integral equations in 3D acoustics," Journal of the Acoustical Society of America, vol. 88, no. 2, pp. 918-937, 1990.

[10] R. A. Jeans and I. C. Mathews, "Solution of fluid-structure interaction problems using a coupled finite element and variational boundary element technique," Journal of the Acoustical Society of America, vol. 88, no. 5, pp. 2459-2466, 1990.

[11] M. Caresta and N. J. Kessissoglou, "Acoustic signature of a submarine hull under harmonic excitation," Applied Acoustics, vol. 71, no. 1, pp. 17-31, 2010.

[12] O. Von Estorff, "Efforts to reduce computation time in numerical acoustics - An overview," Acta Acustica, vol. 89, no. 1, pp. $1-13,2003$.

[13] M. T. Van Nhieu, "An approximate solution to sound radiation from slender bodies," Journal of the Acoustical Society of America, vol. 96, no. 2, pp. 1070-1079, 1994.

[14] M. Utschig, J. D. Achenbach, and T. Igusa, "Reduction to parts: a semianalytical approach to the structural acoustics of a cylindrical shell with hemispherical endcaps," Journal of the Acoustical Society of America, vol. 100, no. 2, pp. 871-881, 1996.

[15] B. Wang, W. Tang, and F. Jun, "Approximate method to acoustic radiation problems: element radiation superposition method," Chinese Journal of Acoustics, vol. 27, pp. 350-357, 2008. 
[16] A. Rosen and J. Singer, "Vibrations of axially loaded stiffened cylindrical shells," Journal of Sound and Vibration, vol. 34, no. 3, pp. 357-378, 1974.

[17] M. Baruch, J. Arbocz, and G. Q. Zhang, "Laminated conical shells: considerations for the variations of the stiffness coefficients," in Proceedings of the 35th AIAA/ASME/ASCE/AHS/ASC Structures, Structural Dynamics, and Materials Conference, pp. 2505-2516, April 1994.

[18] M. Caresta and N. J. Kessissoglou, "Structural and acoustic responses of a fluid-loaded cylindrical hull with structural discontinuities," Applied Acoustics, vol. 70, no. 7, pp. 954-963, 2009.

[19] E. A. Skelton and J. H. James, Theoretical Acoustics of Underwater Structures, Imperial College Press, London, UK, 1997.

[20] M. C. Junger and D. Feit, Sound, Structure, and Their Interaction, MIT Press, Cambridge, UK, 1986. 

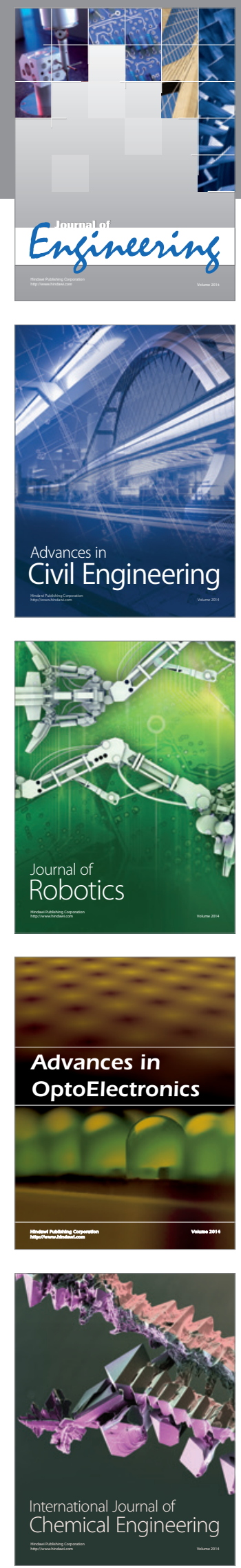

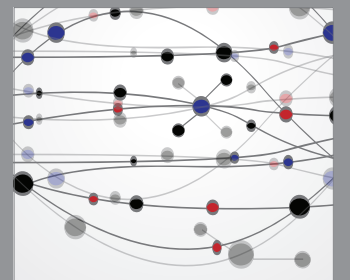

The Scientific World Journal
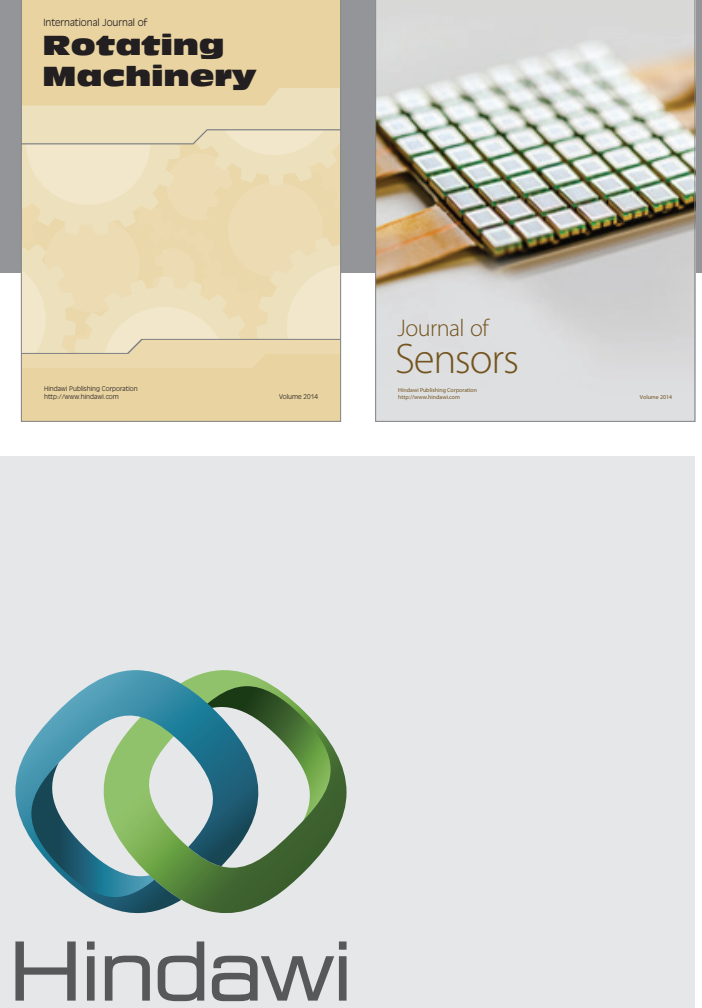

Submit your manuscripts at http://www.hindawi.com
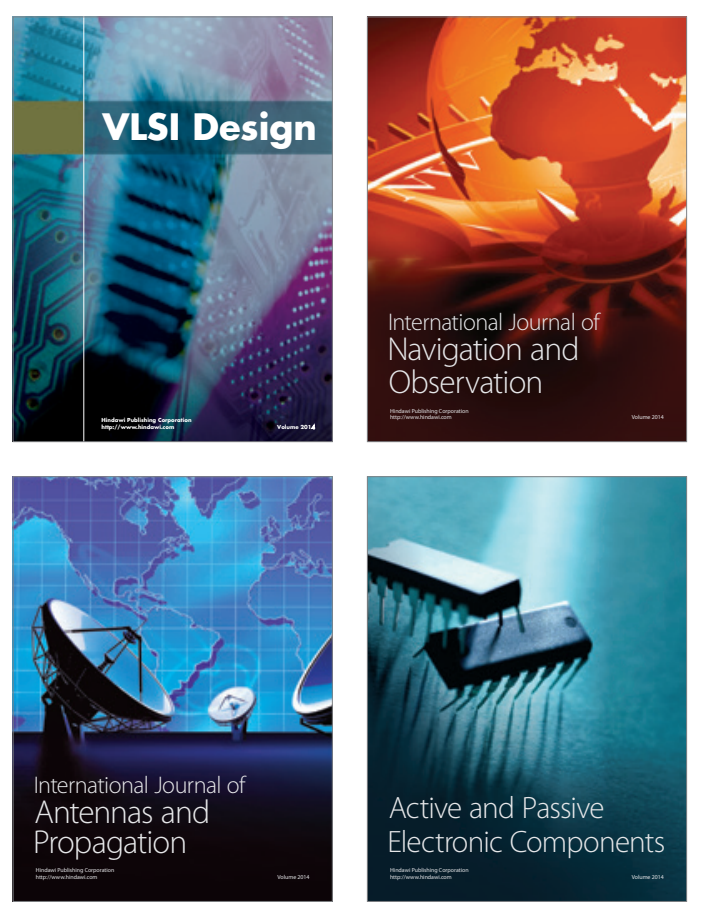
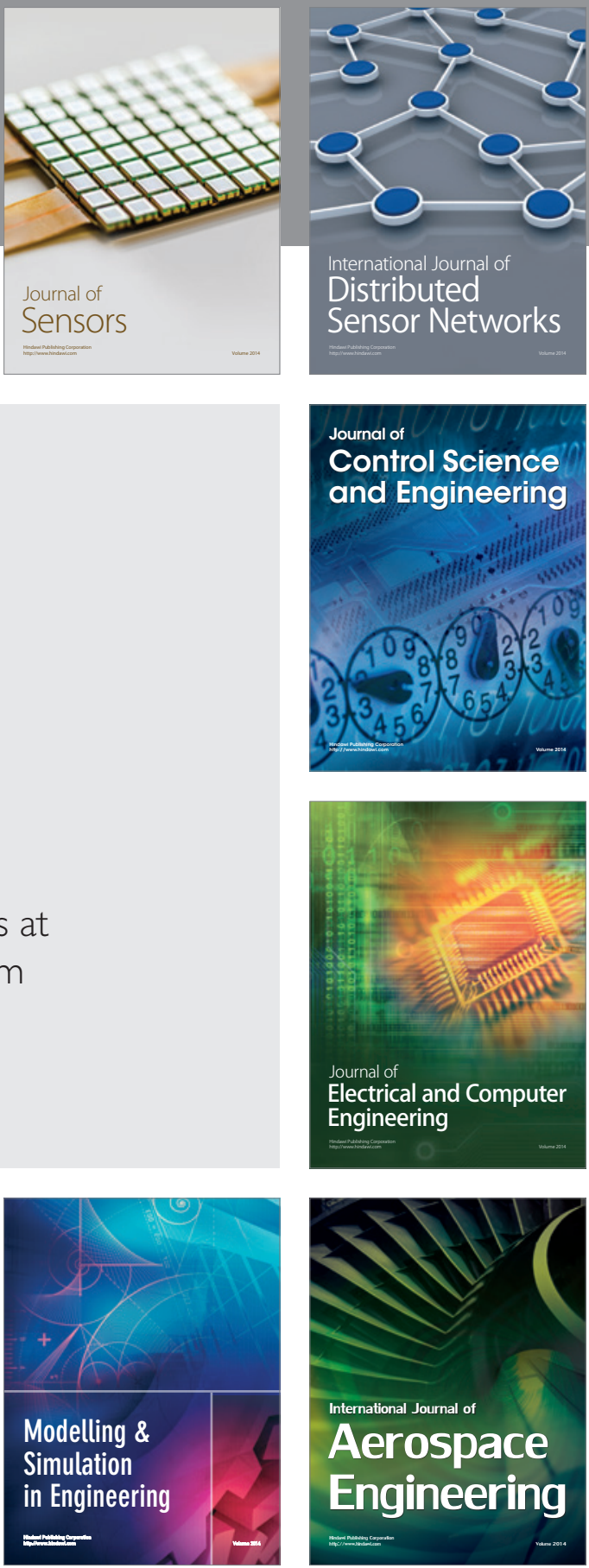

Journal of

Control Science

and Engineering
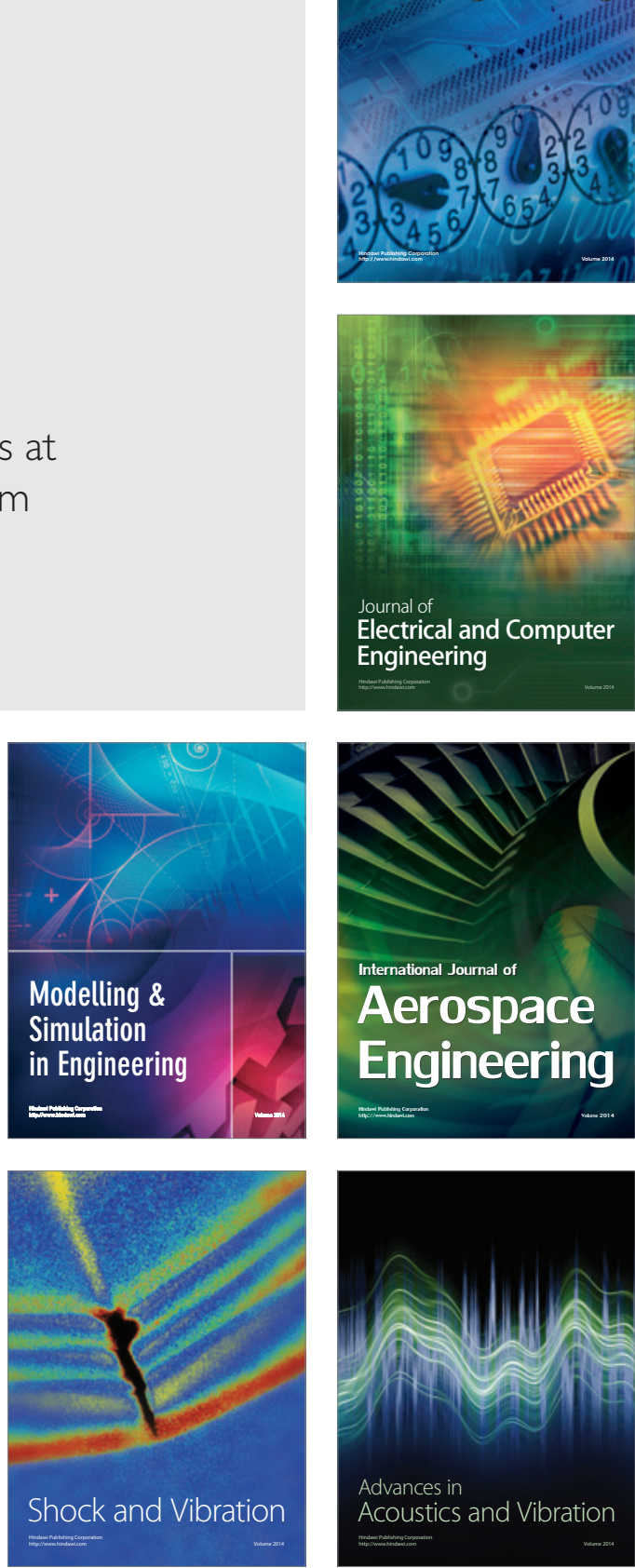